\title{
New Trends in Eyelid Reconstruction
}

\author{
A.Y.Farid, T.H.Olwan, A.M.Abd El-Mofeed and H.A.Abou El-Fadl \\ General and Plastic Surgery Dept., Faculty of Medicine, Benha Univ., Benha, Egypt \\ E-Mail:Heba@gmail.com
}

\begin{abstract}
Although advances in radiotherapy and chemotherapy for malignancies in head and neck have been remarkable, surgical resection followed by reconstructive surgery is still the mainstay of treatment specially in eyelid malignancie. Of the reconstructive systems, microsurgical tissue transfere has been viewed as the standard strategy for reestablishing post employable capacity and morphology in all head and neck zones with the exception of in eyelid remaking as it is considered on of the choices as opposed to the standard one.The recreation stepping stool comprises of different advances beginning from the least complex to the most intricate alternative. When in doubt, when arranging an individual patient's remaking, endeavor the least mind boggling and most secure choice from the reconstructive stepping stool first, while keeping up structure and capacity. Adhering to the standard of supplanting the tissue evacuated with a tissue the same extraordinarily in eyelid territory. The plastic specialist ought to be OK with the full armamentarium of reconstructive procedures, and ought to have the option to choose which strategy is the best for every specific patient and imperfection. Eyelid reproduction is an amazingly requesting process that needs persistent enhancements and refinements. Also, a well information on eyelid life systems is an absolute necessity to accomplish a decent tasteful and useful outcome. Lately, free folds have become the workhorse in head and neck remaking. Despite the fact that it's not the situation in eyelid reproduction, it stays a valuable alternative in remaking of broad complex cases. A skillful reconstructive specialist ought to be comfortable with the armamentarium accessible for reproduction, understanding the focal points and restrictions of every strategy and knowing when and where to receive every one.
\end{abstract}

\section{Introduction}

Eyelid remaking is a difficult issue that faces plastic specialists. Procedures of eyelid recreation change as indicated by the reason. Causes may incorporate awful eyelid misfortune, friendship is either in skin, skin and muscle, or skin, muscle and conjunctiva. These need remaking of skin, muscle and conjunctiva as needs be. Another reason is consume which for the most part influences skin. Since skin of the eyelid is extremely flimsy and uncovered, so profound ignite with ensuing contracture as often as possible happens. Another reason is dangerous tumors in upper or lower eyelid which needs legitimate extraction of the tumor with wellbeing edge with remaking of upper eyelid, lower eyelid, average canthus or sidelong canthus.

The sort of recreation for the eyelids changes as per the kind of imperfection. The upper eyelid is versatile and must be remade working to have the option to move and shield the eye from the external ecological damage. Extraction of upper eyelid skin malignancy regularly prompts moderate to huge imperfection. The point of the remaking is a mobile eyelid, impeccable corneal insurance, great tasteful quality, and adequate sequelae at the benefactor site, which can be accomplished through one-phase fix with three nearby folds: a tarsoconjunctival fold with 2 neighborhood folds [1].

The lower eyelid is steady and non-versatile, so its reproduction must be strong likewise to keep the eye from protruding outside. The deformity that follows extraction of a basal cell carcinoma (BCC) of the lower eyelid can be remade utilizing a pivotal shallow supply route island fold [2]. The auricular chondroperichondral join, utilizing tissue acquired from the concha of the ear (from the back part of the concha), is a generally straightforward careful method and produces insignificant dreariness at the contributor site. Besides, the auricular ligament is effortlessly formed and it forestalls somewhat the postsurgical withdrawal of the skin fold that is picked [3].

Average canthus containing the upper and lower punctum that prompts the lacrimal channel. Its fix whenever harmed is critical to stay away from epiphora.

Recreation of the average or horizontal canthus needs some thoughtfulness regarding remake the sidelong or average palpebral tendon. The sidelong canthus is fundamentally significant for ordinary eyelid form and for remaking of the periorbital district. An unusual horizontal canthus shape has huge ramifications with respect to sidelong visual field and influences periocular cosmoses, and this can be remade by periosteal folds [4].

In different circumstances, remaking of eyelids and attachment in instances of globe evacuation is expected to make the patient ready to wear a counterfeit eye. For instance, for an all-encompassing absolute maxillectomy after a maxillary disease, we can utilize temporalis move for dynamic eye attachment reproduction [5]. We can utilize an island fold on the brow dependent on the anterofrontal part of the shallow worldly supply route for remaking of the eye attachment [6].

The point was to examine techniques for recreation of the eyelids concentrating on the new patterns including microsurgical tissue transfere. Microsurgery have gotten significant in complex cases in eyelid reproduction. The capacity and tasteful outcomes in eyelid remaking rely upon the best possible determination of strategy for fix.

\section{Upper eyelid reconstruction}


The following charts is an overview of Upper eyelid reconstruction Fig $(1,2)$.

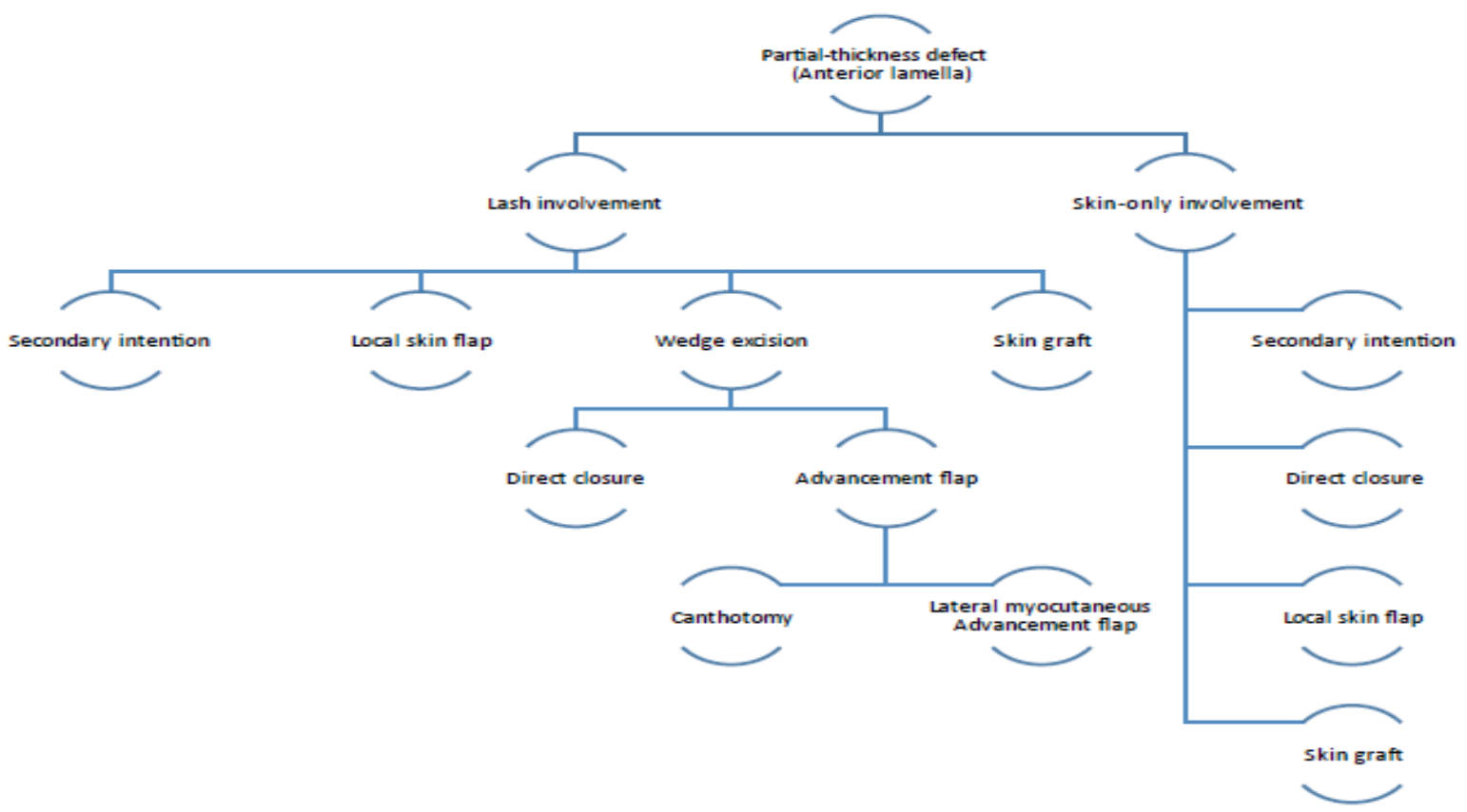

Fig (1) Schematic overview of anterior lamellar defects (Gabriela M. E., Angela M. P., 2016).

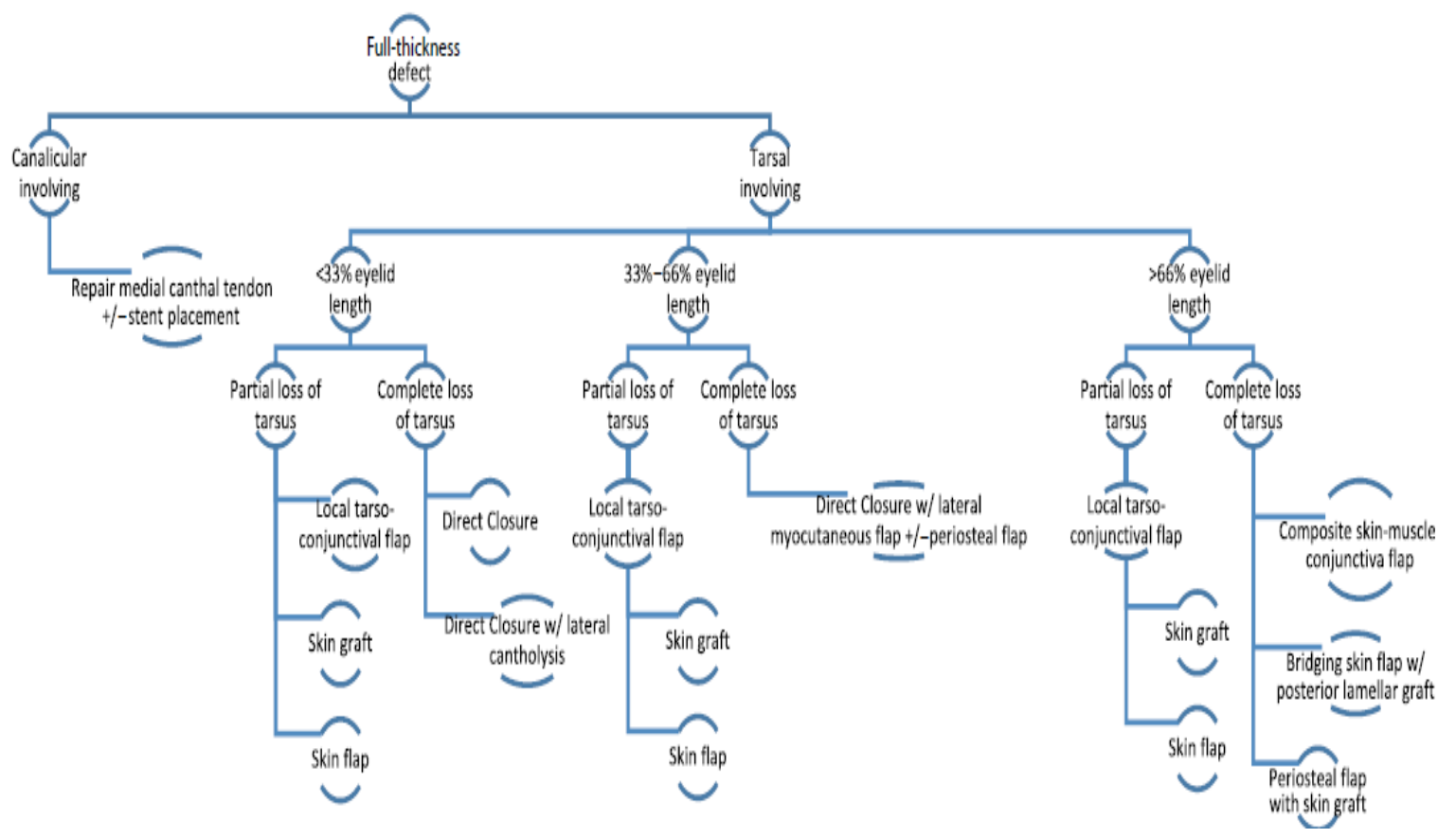

Fig (2) Schematic overview of full-thickness defects. (Gabriela M. E., Angela M. P., 2016).

\section{Direct closure}

An eyelid defect of $25 \%$ or less may be closed directly in most patients. When combined with cantholysis, even a defect occupying up to $50 \%$ or more of the eyelid may be closed directly [7].

Depending on the laxity of the eyelid tissues, full thickness defects of the lid margin involving up to onethird of the horizontal length may be closed with direct closure [8].
Minimal tension can be released with a lateral canthotomy or cantholysis. If superior tarsus remains, completion of a pentagonal resection or complete excision of the residual tarsus may be performed to allow for the ends to meet appropriately [8].

The steps of eyelid margin repair include: 1Reforming the lid margin, 2-Closure of the tarsal plate, 3-Everting the lid margin to prevent notching, 4Closing the skin [8]. 
A

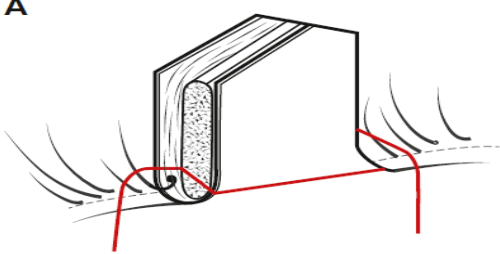

C

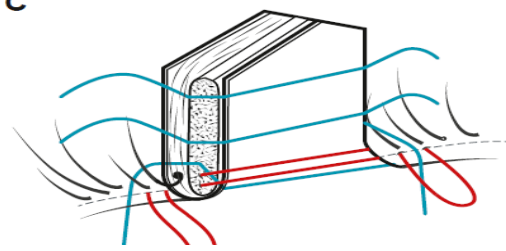

B

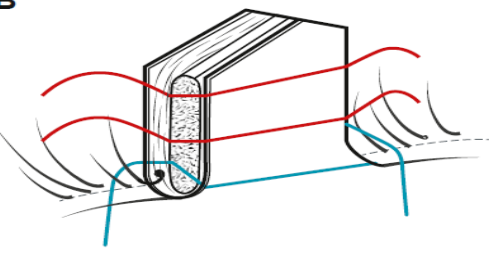

D

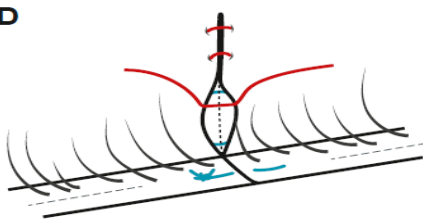

Fig (3) Lid margin repair. (A) Align the lid margin. (B) Suture the superior tarsal plate for stabilization. (C) Evert the marginal wound edge in a vertical mattress fashion. (D) Close the overlying skin [8].

\section{Grafts in upper eyelid reconstruction}

Foremost lamellar imperfections can be reproduced with a full-thickness skin unite [9].

Perfect contributor destinations incorporate abundance upper and lower eyelid skin and back auricular, preauricular, or supraclavicular skin. Split thickness skin graftsshould be kept away from. [10].

Tarso-conjunctival unions are a phenomenal decision for back lamellar recreation, following the worldview of supplant like-with-like. They are collected from the upper eyelid, leaving in any event 3 to 4 mmof distal bone structure to maintain a strategic distance from upper cover contortion. The benefactor site mends by optional aim. Astounding outcomes have been accounted for utilizing tarso-conjunctival unions for fixing imperfections of up to 75 percent of the eyelid length [11].

As of late, a pre-assembled composite join utilizing auricular ligament and buccal mucosa for back lamella reproduction was demonstrated to be effective [12].

Jianjun et al, conceived a composite join of acellular human dermis and split-thickness buccal mucosa to supplant the back lamella of the eyelid [13].

3. Neighborhood Flaps for Subtotal and Total Upper Eyelid Reconstruction

Reproduction of upper eyelid deformity is mind boggling, in light of the fact that, in contrast to the lower eyelid, there isn't sufficient accessible tissue around it and protection of capacity and shape is all the more requesting to accomplish [14].

Numerous methodology have been accounted for upper eyelid recreation; these strategies incorporate the various composite eyelid unites [15], chondromucosal fold, the Cutler-Beard fold from the lower eyelid [16], the poorly based tarso-conjunctivalflap [17].

Late writing concentrated on less obtrusive methods dependent on the utilization of inherent tissues of the upper eyelid and periorbital district. In 1997, Okada proposed the utilization of a level V-Y myotarso-cutaneous progression fold for enormous upper eyelid surrenders up to $60 \%$ of its width [18]. This method was effectively imitated by Rosa, in 16 cases [19].

Demir applied the $\mathrm{V}-\mathrm{Y}$ way to deal with a myocutaneous progression fold collected from the preseptal segment of the upper eyelid. This work misused the skin overabundance of the maturing upper eyelid yet as we would like to think brought about deficiently situated scars [20].

In 2003, Irvine first proposed the utilization of a headway fold from the lingering homolateral upper eyelid for full thickness recreations [21].

Recently, a few creators have proposed various endeavors to consolidate a stylish blepharoplasty way to deal with eyelid imperfection fix. Hatoko in 1999 right off the bat depicted a strategy for recreation of a full-thickness lower eyelid imperfection utilizing overabundance skin of the lower eyelid with a hard sense of taste mucosal unite [22].

Most dependable strategies in upper eyelid reproduction as indicated by [3]:

Even V-Y Myo-tarso-cutaneous Advancement Flap.

The V-Y myo-tarso-cutaneous fold is performed for upper eyelid deserts up to $60 \%$ of eyelid width. It is structured on the parallel canthal district, pedicled on orbicularis oculi and reaching out at the crow's feet in a "V" design. The average side of the fold compares to parallel edge of the deformity; the prevalent edge follows the line of the unrivaled palpebral crease. The fold stature to-width proportion can arrive at 1:4 [24]. To build fold's portability, it could be important to play out a sidelong canthotomy [19]. 


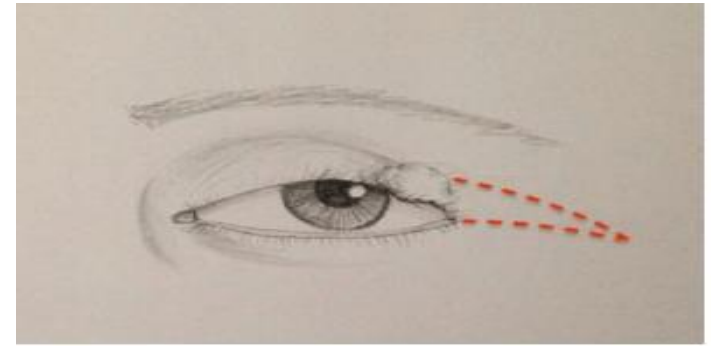

(a)

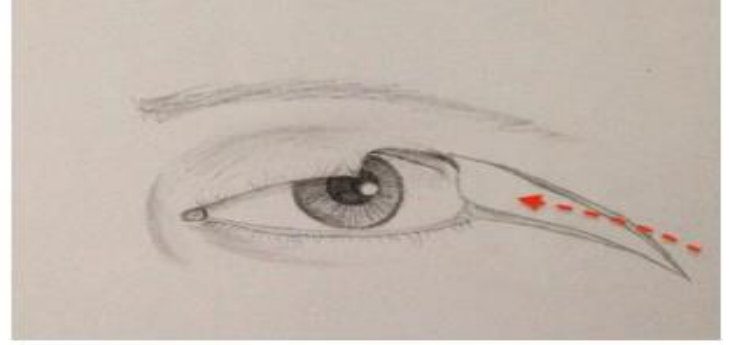

(b)

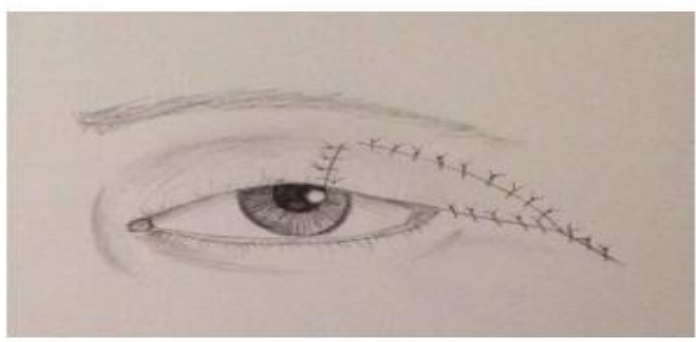

(c)

Fig (4) The V-Y myo-tarso-cutaneous flap. Flap is designed on the lateral canthal region (a). Tumor excision, the medial side of the flap corresponds to lateral margin of the defect, and the superior edge follows the line of the superior palpebral fold (b). Flap advancement and donor-site closure in a V-Y fashion (c) [23].

\section{Anterior lamella reconstruction}

At the point when the reconstructive specialist needs to confront a fractional thickness imperfection, front lamella, the utilization of orbicularis oculi myocutaneous folds structured in different styles has a more noteworthy tasteful result contrasted and full-thickness skin joins. Utilizing a fold gives a superior surface and shading match with neighboring tissues and guarantee less compression during mending. Upper eyelid remaking can be performed with Fricke fold. These rendering or revolution headway folds can be joined with a tarso-conjunctival unite for back lamellar remaking in the event of full-thickness imperfection [23].

\section{The fricke flap}

It is valuable in the monocular patients that the impediment of the visual pivot with strategies, for example, the Hughes or Cutler-Beard folds would be unsuitable. The viability and unwavering quality of the strategy are possibly improved when utilized in mix with quick intraoperative tissue development [25].

\section{Frontalis muscle flap}

Frontalis muscle fold is a reconstructive choice for subtotal and all out upper eyelid deformity. It is raised from the previous imperfection, therefore dodging an extra temple scar. The back lamellar recreation is made by palatal mucoperiosteal unite [25].

After the back lamella is recreated, the levator palpebrae is reinserted and sewed to the periosteum of the join. The analyzation is helped through the previous palpebral deformity in a pre-septal plane over the supraorbital edge [25].

This dismemberment at that point arrives at a subcutaneous plane over the frontalis muscle. A caudally pedicled frontalis muscle fold was then raised and diverted down to stretch out from the better orbital edge than the recreated eyelid edge. The fold was custom-made to coordinate the imperfection without pressure and stitched to the injury edges. A fullthickness skin unite is utilized to supplant the upper eyelid skin.

The principle bit of leeway of this method is the nonappearance of scar at the contributor site [25].

- Posterior Lamella Reconstruction:

Chondro-mucosal Flap for Total and Subtotal Upper Eyelid Reconstruction.

Chondro-mucosal fold is a reconstructive choice for the back lamella; the fold is structured along the sidelong nasal divider and depends on the terminal part of the ipsilateral dorsal nasal corridor. It is a pivotal chondro-mucosal fold secured with a skin unite for the front lamella [23].

The foremost lamella is reproduced with a skin unite reaped from the contralateral upper eyelid. A blepharorrhaphy is performed to upgrade skin join take and stay away from withdrawal of recreated eyelid. The benefactor site deformity is fixed with direct conclusion or left mend precipitously [14]. 


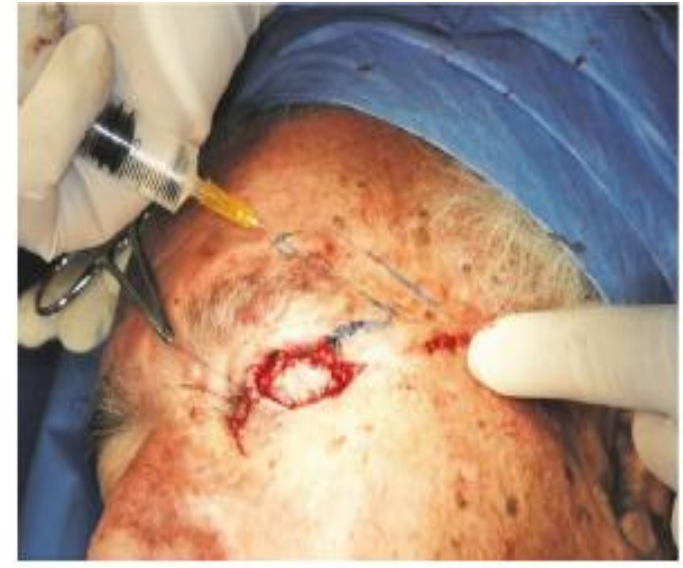

(a)

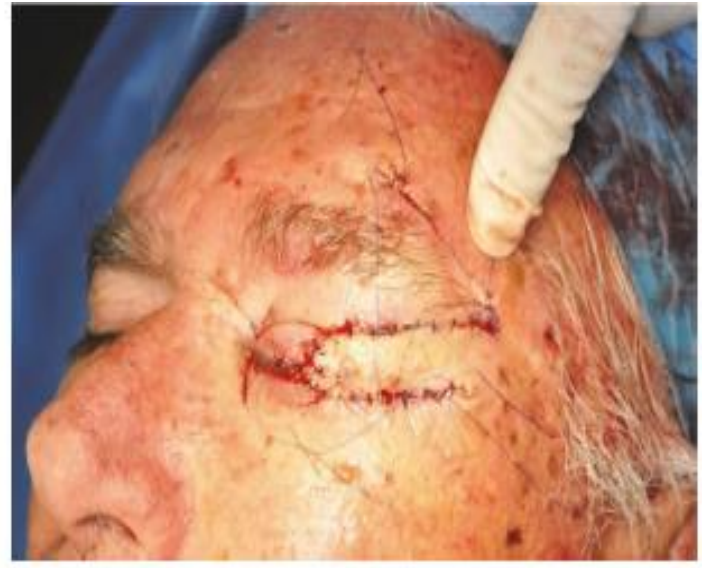

(b)

Fig (5) Fricke flap for subtotal upper eyelid reconstruction (anterior lamella). Intraoperative marking of Fricke flap, the posterior lamella has already been reconstructed using a buccal mucosa graft (a); the transposition flap and donor site are sutured (b) [23].

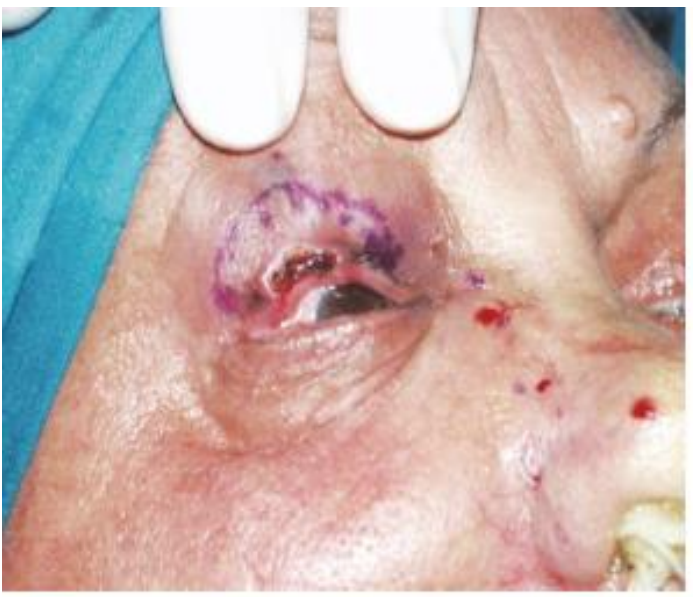

(a)

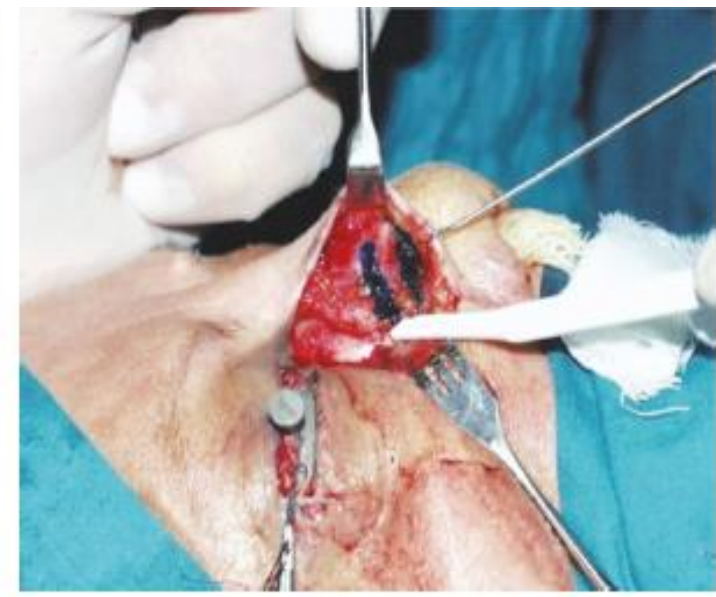

(b)

Fig (6) Chondro-mucosal flap. (a)Preoperative picture, tumor excision is going to involve the whole width of upper eyelid. (b)Intraoperative marking of chondro-mucosal flap, lateral nasal wall [23].

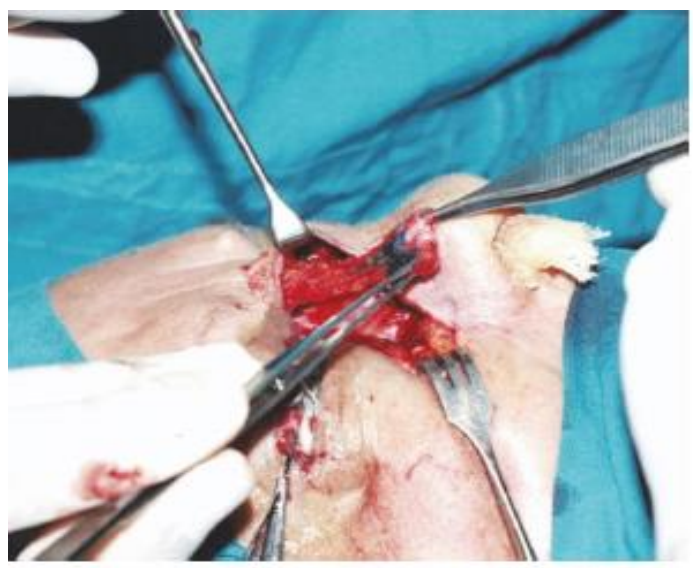

(a)

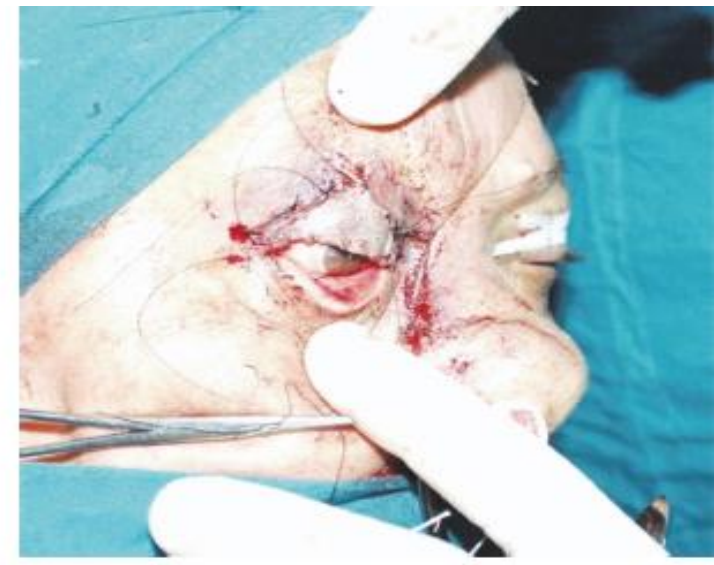

(b)

Fig (7) Chondro-mucosal flap. (a)The flap is raised on his pedicle. (b)The flap is covered with a full-thickness skin graft [23]. 


\section{Cutler-beard flap}

The Cutler-Beard lid-sharing flap is a skin muscleconjunctival flap harvested from the lower eyelid and advanced to cover upper eyelid defect up to $100 \%$ in width.
The main drawback of this procedure is the lack of a rigid posterior lamella which can lead to instability of the reconstructed eyelid; free tarso-conjunctival grafts or hard palate mucosa grafts can be used to provide support to the flap (after flap inset) [26].

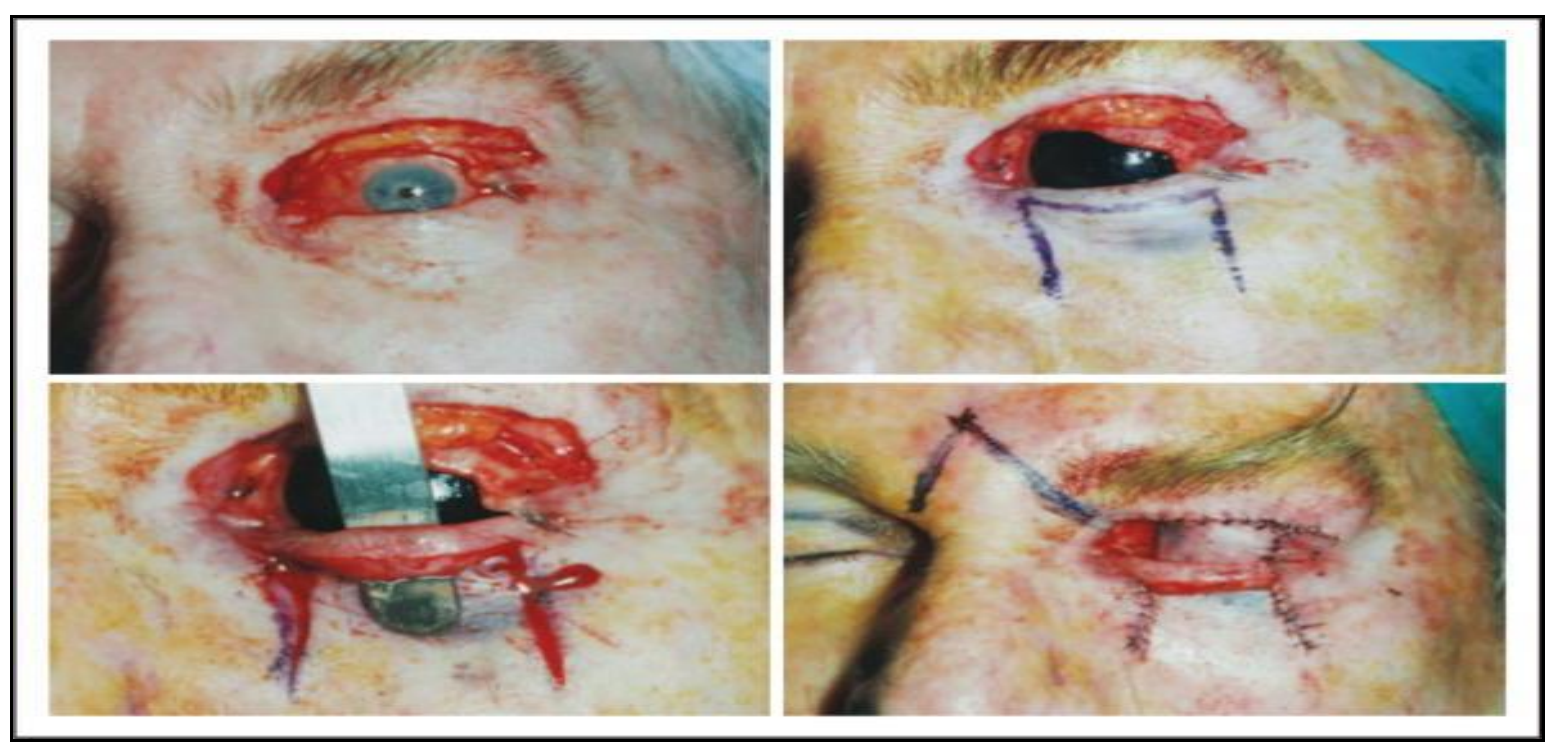

Fig (8) The Cutler-Beard Bridge flap used to repair large full thickness defects of the upper lid [27].

A replacement of upper eyelid tarsus with Achilles tendon "as an allograft" as part of the Cutler-Beard procedure was successful in repairing large upper eyelid defects. The human Achilles tendon is composed of collagen fibril bundles, arranged in parallel, with supportive cells in a dense connective tissue. It has a measurable elasticity, viscosity, and plasticity and reacts to functional demands [28].

The modified Cutler-Beard procedure with the autogenous ear cartilage for tarsal reconstruction was

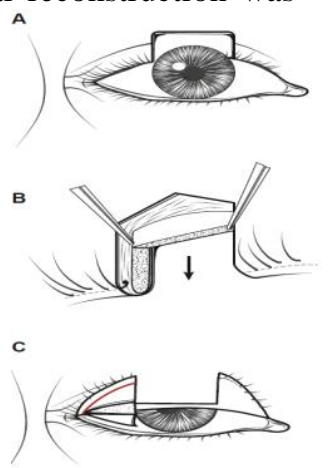

proposed by Salil et al, 2016, which is an acceptable procedure for repair of eyelid defects of $70-100 \%$. In addition to providing satisfactory cosmetic results, it is safe and cost effective [29].

\section{Pedicled lower lid Sharing flap Sliding tarso-conjunctival flap}

The sliding tarso-conjunctival flap is a transposition flap based on adjacent conjunctival defects involving the medial or lateral upper eyelid [8].
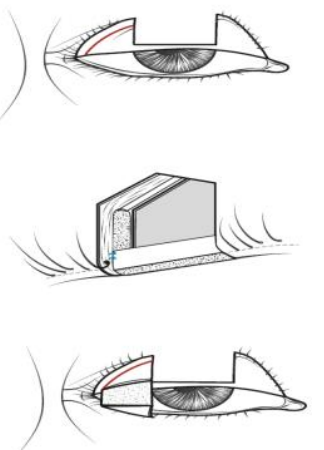

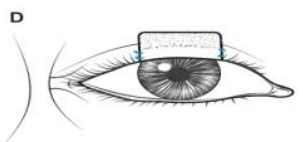

Fig (9) Local tarso-conjunctival flap. (A) When full height of tarsus missing (left), evert the eyelid and incise superior tarsus of remaining eyelid (right) leaving behind a 4-mm margin for stability. (B) When at least $4 \mathrm{~mm}$ of superior tarsus remains in a full-thickness defect (left), advance the remaining tarsus to the margin (right). (C) Create a tarso-conjunctival flap by dissecting the anterior tissue off the flap (left) and create relaxing incisions in the conjunctiva superiorly (right). (D) Transpose the tarsoconjunctival flap into the defect to reform the margin [8]. 


\section{Blepharoplasty flap}

As of late, two creators have revealed the use of the blepharoplasty skin entry point to reconstructive medical procedure of the upper eyelid. In 2008, Then et al. portrayed a better pivoted blepharoplasty fold than activate palpebral excess skin to cover an average imperfection following extraction of average injury in upper eyelid. This methodology is suggested distinctly for average imperfections not including the free fringe [30].

With respect to including the pre-septal piece of upper eyelid, in 2014, Takahashi proposed to incorporate the skin deformity after malignancy extraction in a blepharoplasty design. This strategy misuses the skin excess typically expelled during blepharoplasty, however it is just appropriate for preseptal imperfections [31].

A. Bulla et al, 2017, recommended that the back lamella is fixed with a tarso-conjunctival headway fold, constraining the sign to patients with $3-4 \mathrm{~mm}$ of lingering bone structure. The outcomes show that the progression fold is better sketched out in the upper eyelid blepharoplasty example to keep away from intricacies, for example, lagophthalmos. Additionally, the sign of the method is reached out to deserts including the whole vertical width of bone structure by utilizing a Chondro-mucosal join. This limited inward lamella withdrawal and keratopathy. [32].

This method can be viewed as one of the principal approaches consolidating the tasteful blepharoplasty example to upper eyelid remaking. This methodology exploits this skin repetition to fix imperfections of the focal third of the upper eyelid from the minor zone up to the palpebral wrinkle. In a perfect world, the procedure is most appropriate for injuries on the center

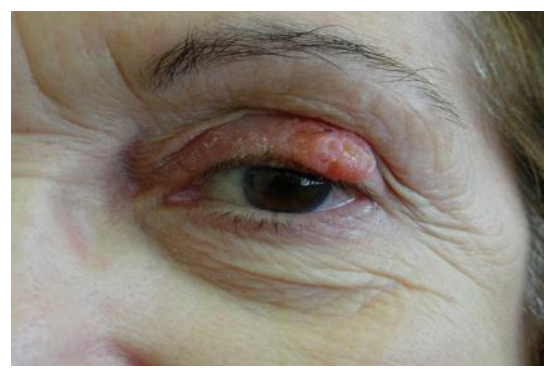

$a$

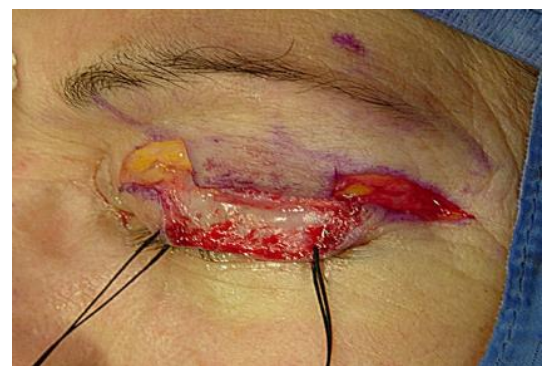

third of the upper eyelid, at a greatest separation of 1 $\mathrm{cm}$ from the free fringe [32].

A muco-chondral join from the nasal septum is liked to remake the back lamella. As the attributes of this unite best match those of the back lamella, for example, bolster capacity and versatility. All things considered, this strategy could be matched to a join reaped from some other chose site [32].

The reconstruction of a total eyelid defect is intricate because of the lack of surrounding available tissue of matching color and texture.. The Mustardé method is the most common approach for total and full thickness upper eyelid reconstruction. However, the defect size that can be reconstructed using the lower eyelid is limited, and a second surgery is required to separate the flap [34].

\section{Upper eyelid reconstruction and micro surgery}

Kushima et al. reported a case of total upper eyelid reconstruction using a free forearm flap for a traumatic upper eyelid defect [34]. In 2007, Ueda et al. reported functional reconstruction of the upper eyelid using a suspension technique that involved securing the palmaris longus tendon of the forearm flap to the frontalis muscle [35]. For functional eyelid reconstruction, it should be considered not only opening but also closing of the eyelid. To treat a total upper eyelid defect, Hiroyuki Iwanaga et al. applied a radial forearm-palmaris longus teno-cutaneous free flap, and a split palmaris longus tendon was secured to the frontalis muscle and orbicularis oculi muscle to achieve functional movement in two patients. The purpose of their report was to introduce their new technique of functional upper eyelid reconstruction [36].

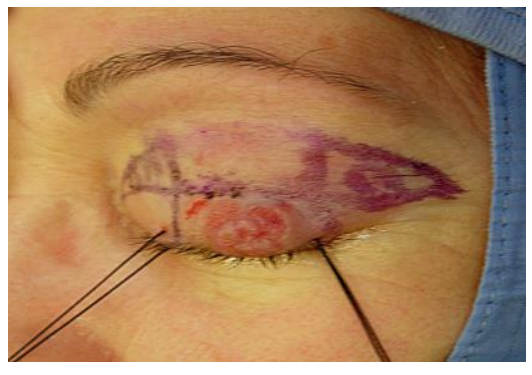

$\boldsymbol{B}$

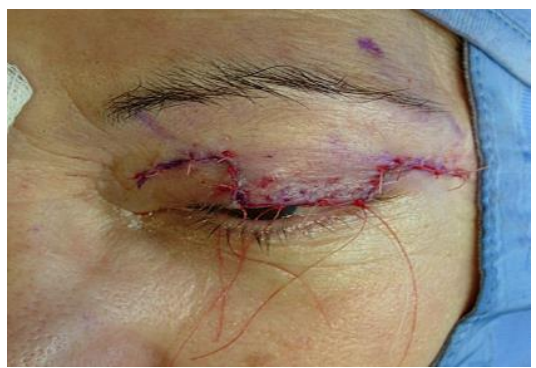

$D$

Fig (10) a A 68-year-old patient with nodular Bowen's disease of left upper eyelid, b Preoperative markings, c Intraoperative view of the defect after tumor excision with exposure of the posterior lamella and flap harvest, d Flap advanced to cover the defect [32]. 


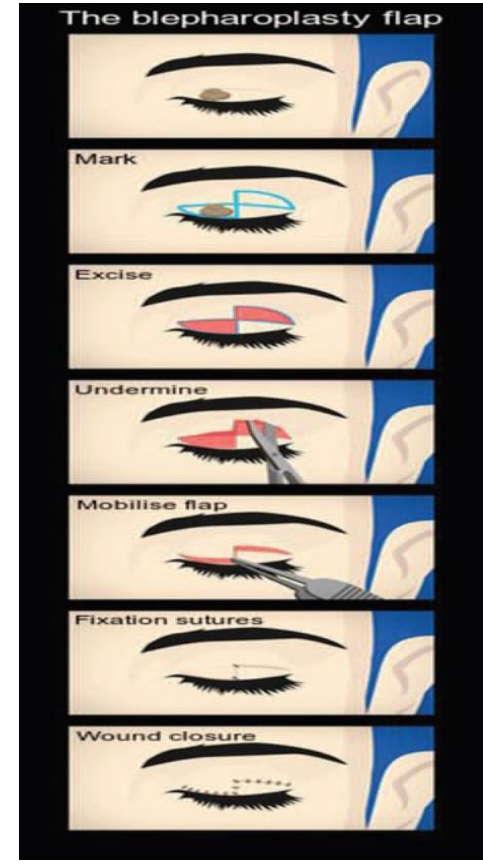

Fig (11) Steps to the blepharoplasty flap.

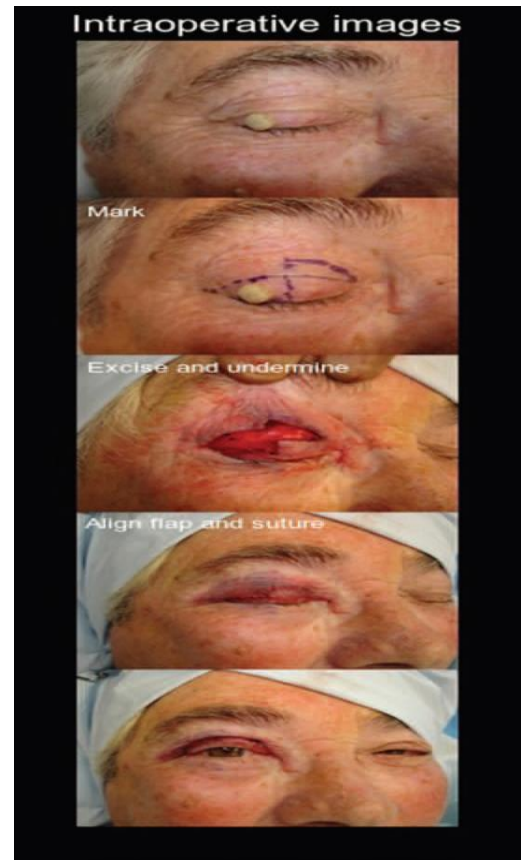

Fig (12) Intraoperative images [33].

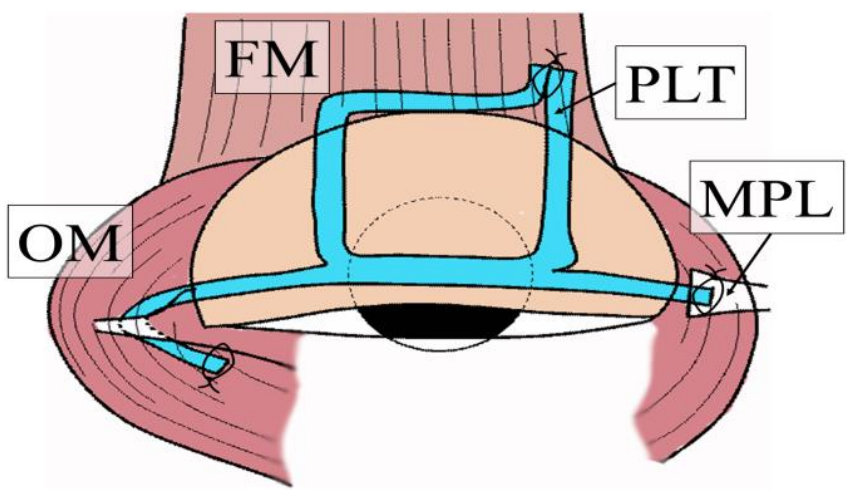

Fig (12) The palmaris longus tendon was split into two strips. The upper strip was fixed to the frontal muscle, and the lower strip was fixed to the medial palpebral ligament and orbicularis oculi muscle. FM, frontal muscle; MPL, medial palpebral ligament; OM, orbicularis oculi muscle; PLT, palmaris longus tendon [36].

The lower arm fold is regularly adaptable and meager; in this manner, it is generally perfect for reproduction of the upper eyelid. In any case, the greater part of the fold may make issues accomplishing these capacities. Kushima et al. attempted to accomplish the initial capacity of the remade upper eyelid utilizing the levator muscle [34].

Be that as it may, the remade eyelid was withdrawn around $5 \mathrm{~mm}$ and the levator palpebrae superioris muscle can't withdraw the lower arm fold. In 2003, Ueda et al. detailed the suspension procedure that included making sure about the palmaris longus ligament of the lower arm fold to the frontalis muscle [35].

The Friedenwald-Guyton strategy includes the bone structure suspension method. With Hiroyuki Iwanaga et al. method, the ligament is made sure about to the frontalis muscle by means of free belt through the square subdermal burrow. The closures of the belt are integrated, and the length of the sash can be balanced by delivering or fixing the ligament after medical procedure. With this method, the palmaris longus ligament was part into two strips, and the upper strips were made sure about to the frontalis muscle through the square subdermal burrow [36].

The orbicularis oculi muscle around the palpebral crevice has a significant job in shutting the eyelids. To accomplish muscle work, muscle progression and strain are required. In this way, to accomplish the end capacity of the reproduced eyelid, congruity of the muscle ring ought to be kept up [36].

They made sure about the lower segment of the split palmaris longus ligament to the orbicularis oculi muscle. Compression of the remainder orbicularis oculi muscle moves the palmaris longus ligament, which shuts the eyelids [36].

At times, all layers of the upper eyelid may be lost after resection. Microsurgical reconstructive methods 
have reformed post ablative recreation. Be that as it may, there is a cutoff to recreating the dynamic elements of the eyelid utilizing just free fold move. The opening and shutting elements of the eyelids are significant for looking after vision. Hiroyuki Iwanaga et al. procedure is a mix of the most recent method utilizing the spiral lower arm palmaris longus tenocutaneous free fold joined with the cover suspension strategy [36].

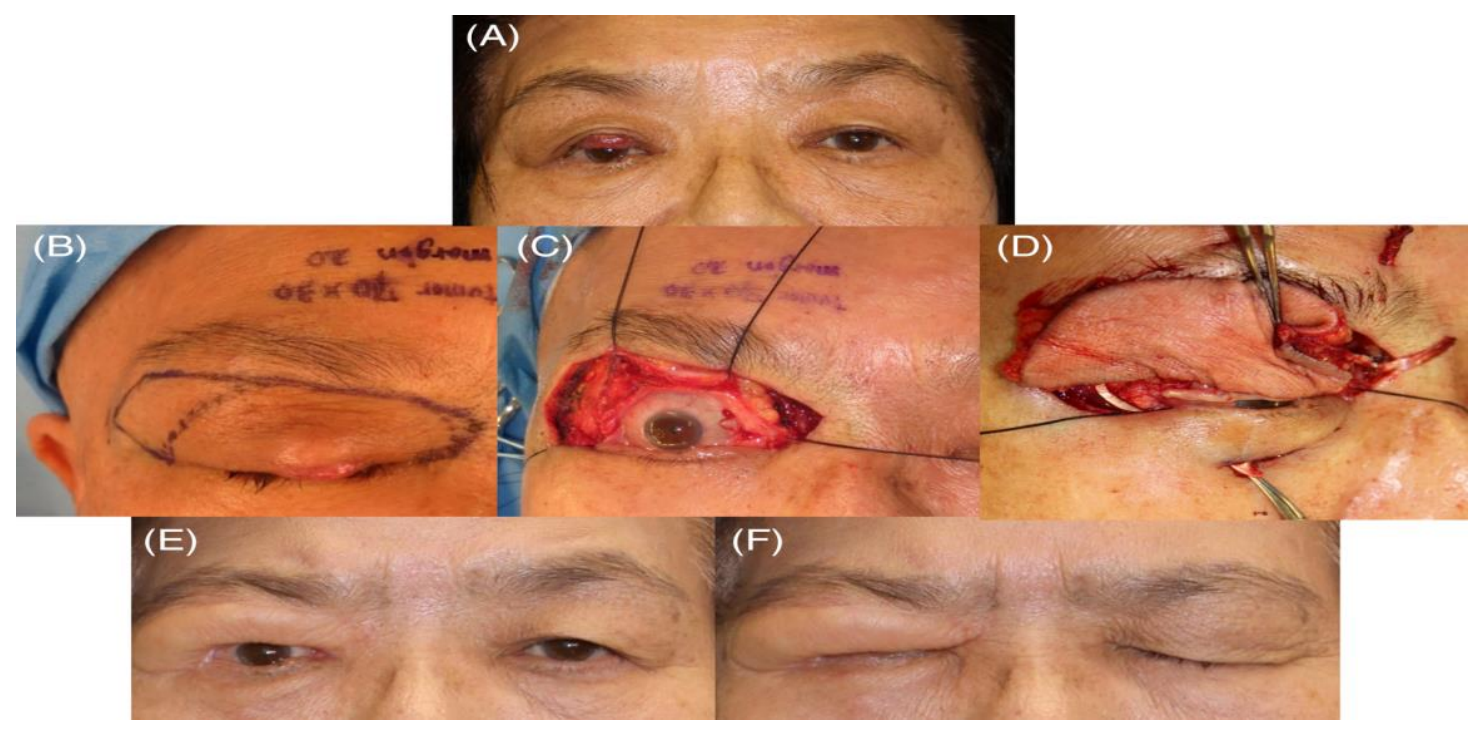

Fig (13) A, Merkel cell carcinoma in the right upper eyelid. B, Excisional margins. C, The right upper eyelid tumor was resected. An $80 \times 40 \mathrm{~mm}$ full-thickness upper eyelid defect. D, The medial lower strip was secured to the medial palpebral ligament and the lateral lower strip was secured to the lower orbicularis oculi muscle through the lateral canthal ligament. E, Postoperative lid opening. F, Postoperative lid closing (4 years after surgery) [36].

\section{Conclusion}

Reconstruction of eyelids needs to be familiar with the anatomy of the eyelid including the characters of the skin, the muscle affecting function of eyelid, the supportive structures as septa, tarsal plate and ligaments, and the glans present in the eyelid, and the need to preserve it for normal or near normal function of this lid.

\section{References}

[1] J.Yan, L.Liu, J.Quin. Reconstruction of upper eyelid and medial canthus following basal cell carcinoma resection: a successful one stage repair with three local flaps: International Journal of Dermatology, Vol.52, PP.611, 2013.

[2] Z.Yan, Z.Jianhui, W.Xiaoyan. The application of axial superficial temporal artery flap for repairing the defect secondary to the removal of the lower eyelid basal cell carcinoma: British Journal of Oral and Maxillofacail Surgery, Vol. 52, PP.72, 2014.

[3] O.Rivas, C.Blanco, G.Sixto. Auricular ChondroPerichondreal Graft in the Reconstruction of the lower Eyelid: ACTAS Dermo-Sifiliograficas, Vol.105, PP.307, 2013.

[4] A.Y.Nemet. The lateral canthus web and its surgical management: Journal of Plastic, Reconstructive and Aesthetic Surgery, Vol. 67, PP.480, 2014.

[5] H.Motomura, T.Hatano. R.Kobayashi. Dynamic eye socket reconstruction after extended total maxillectomy using temporalis transfer: Journal of plastic, reconstructive and aesthetic surgery, Vol.67, PP.78, 2013.

[6] N.Omori, N.Mitsukawa, Y.Kubota. Reconstruction of contracted eye socket using an anterofrontal superficial temporal artery island flap and scapha composite grafting in an elderly patient: Journal of plastic, Reconstructive and Aesthetic surgery, Vol.65, PP.1722, 2012.

[7] A.C.Suryadevara , K.S.Moe. Reconstruction of Eyelid Defects," Facial Plastic Surgery Clinics of North America, Vol. 17(3), PP.419-428, 2009.

[8] M.E.Gabriela, M.P.Angela. Upper Eyelid reconstruction, Facial plastic surg Clin Am, Vol. 42, PP.173-182, 2016.

[9] N.Shorr . "Upper eyelid skin grafting: an effective treatment for lagophthalmos following blepharoplasty.," Plastic and Reconstructive Surgery. Vol. 112(5), PP.1444-1448, 2003.

[10] H.Kakizaki. "Oculoplastic surgery for lower eyelid reconstruction after periocular cutaneous carcinoma," International Ophthalmology Clinics, Vol. 49(4), PP.143-155, 2009.

[11] M.J.Hawes, A.S.Grove, E.M.Hink. "Comparison of free tarso-conjunctival grafts and hughes tarsoconjunctival grafts for lower eyelid reconstruction," Ophthalmic Plastic \& Reconstructive Surgery, Vol. 27(3), PP.219-223, 2011. 
[12] G.Nicoletti, O.Jaber, M.M.Tresoldi. Prefabricated composite graft for eyelid reconstruction. Facial Plast Surg, Vol. 5, PP.534-538,2015.

[13] G.u.Jianjun, Jiaje Zhai, Jiaqi Chen. The use of acellular human dermis composite graft for upper eyelid reconstruction in ocular injury, $\mathrm{J}$ trauma, Volume, Vol. 72, PP.288-292, 2012.

[14] N.Scuderi . "Total and subtotal upper eyelid reconstruction with the nasal chondro-mucosal flap: A 10-year experience," Plastic and Reconstructive Surgery. Vol. 115 (5), PP.12591265, 2005.

[15] C.K.Beyer-Machule, A.Shapiro, B.Smith, "Double composite lid reconstruction: A new method of upper and lower lid reconstruction, Ophthalmic Plastic and Reconstructive Surgery, Vol. 1, PP.97, 1985.

[16] N.L.Cutler , C.Beard. "A method for partial and total upper lid reconstruction," American Journal of Ophthalmology, Vol. 39 (1), PP.1-7, 1955.

[17] J.A.Mauriello, R.Antonacci. "Single tarsoconjunctival flap (lower eyelid) for upper eyelid reconstruction ('reverse' modified hughes procedure)," Ophthalmic Surgery, Vol. 25 (6), PP.374-378, 1994.

[18]E.Okada, Y.Iwahira, Y.Maruyama. The V-Y advancement myotarsocutaneous flap for upper eyelid reconstruction. Plast Reconstr Surg, Vol. 100(4), PP.996-998, 1997.

[19] J.Rosa, D.Casal, P.Moniz. Upper eyelid reconstruction with a horizontal $\mathrm{V}-\mathrm{Y}$ myo-tarsocutaneous advancement flap. J Plast Reconstr Aesthet Surg JPRAS, Vol. 63(12), PP.2013-,2017.

[20]Z.Demir, S.Yuce, S.Karamursel. Celebioglu, "Orbicularis oculi myo-cutaneous advancement flap for upper eyelid reconstruction," Plastic and Reconstructive Surgery. Vol. 121(2), PP.443-450, 2008.

[21]F.Irvine, A.A.McNab. A technique for reconstruction of upper lid marginal defects. Br J Ophthalmol.vol 87(3), PP.279-281, 2003.

[22] M.Hatoko. Reconstruction of full-thickness lower eyelid defects using a blepharoplasty technique with a hard palate mucosal graft. Ann Plast Surg, Vol. 42(6), PP.688-692, 1999.

[23] Federico Lo Torto, Luigi Losco, Nicoletta Bernardini, Manfredi Greco, Gianluca Scuderi, and Diego Ribuffo, Surgical Treatment with Locoregional Flaps for the Eyelid: A Review, Hindawi BioMed Research International, 2017

[24] G.Wilcsek . "The 'RITE' use of the Fricke flap in periorbital reconstruction," Eye. Vol. 19, no. 8, PP.854-860, 2005.
[25] R.D.Jean, W.W.Wong, M.C.Martin."Single-stage frontalis muscle flap for full-thickness reconstruction of the upper eyelid," The Journal of Craniofacial Surgery. Vol. 22(5), PP.1762-1764, 2011.

[26] J.Ahmad, D.W.Mathes, K.M.Itani. Reconstruction of the eyelids after Mohs surgery. Semin Plast Surg, Vol. 22, PP.306-318, 2008.

[27] M.Alghoul, S.J.Pacella, W.T.McClellan. Eyelid reconstruction. Plast Reconstr Surg, Vol. 132, PP.288e, 2013.

[28]L.Erin, D.Keith. Carter, Modification of the Cutler- Beard Procedure Using Donor Achilles Tendon for Upper Eyelid Reconstruction, Ophthalmic Plastic and Reconstructive Surgery. Vol. 21(4), pp 267-270, 2005.

[29] Salil kumar Mandal, James Christian Fleming, Shilpa Gillella Reddy, Brian T, and Fowler, Total upper Eyelid reconstruction with Modified CutlerBeard Procedure Using Autogenous Auricular Cartilage, Journal of clinical and diagnostic research.Vol 10, 2016.

[30] S.Y.Then, R.Malhotra. Superiorly hinged blepharoplasty flap for reconstruction of medial upper eyelid defects following excision of xanthelasma palpebrum. Clin Exp Ophthalmol, Vol. 36(5), PP.410-414, 2008.

[31] Y.Takahashi. Upper eyelid reconstruction by making a skin defect similar to skin removal in blepharoplasty. J Craniofac Surg, Vol. 25(5), PP.e445-e446, 2014.

[32]A.Bulla. A New Approach to Upper Eyelid Reconstruction, Aesth Plast Surg, Vol. 41, PP.346-351, 2017.

[33] Katherine Anne McVeigh , Richard Caesar. Upper Eyelid Reconstruction Using a Blepharoplasty Flap, Ophthal Plast Reconstr Surg. Vol. 33, PP.2, 2017.

[34] H.Kushima, S.Yuzuriha, S.Kondo. Reconstruction of an inner layer defect of the upper eyelid with avulsion of the superior levator palpebrae muscle and orbital fat. Annals of Plastic Surgery, Vol. 51, PP.321-314, 2003.

[35] K.Ueda, S.Oba, M.Okada. Eyelid reconstruction with a composite radial forearm palmaris longus tendon flap. Journal of Plastic, Reconstructive \& Aesthetic Surgery, Vol. 60, PP.256-259, 2007.

[36] Hiroyuki Iwanaga, Takashi Nuri, Masashi Okada, Koichi Ueda. Functional reconstruction of total upper eyelid defects with a composite radial forearm-palmaris longus teno-cutaneous free flap: A report of two cases, Microsurgery, Vol. 39, PP.559-562, 2019. 